\title{
Cumulant-based Parametric Multichannel FIR System Identification Methods $\uparrow$
}

\section{by M. TANKUT ÖZGEN}

Middle East Technical University, Department of Electrical Engineering, Ankara, Turkey

\section{SALEH A. ALSHEBEILI}

King Saud University, Department of Electrical Engineering, Riyadh 11421, Saudi Arabia

\section{A. ENIS ÇETIN}

Bilkent University, Department of Electrical Engineering, Bilkent, Ankara, 06533, Turkey

and A. N. VENETSANOPOULOS

University of Toronto, Department of Electrical Engineering, Toronto, Canada MSS 1 A4

ABSTRACT: In this paper, "least squares" and recursive methods for simultaneous identification of four nonminimum phase linear, time-invariant FIR systems are presented. The methods utilize the second-and fourth-order cumulants of outputs of the four FIR systems of which the common input is an independent, identically distributed (i.i.d.) non-Gaussian process. The new methods can be extended to the general problem of simultaneous identification of three or more FIR systems by choosing the order of the utilized cumulants to be equal to the number of systems. To illustrate the effectiveness of our methods, two simulation examples are included.

\section{Introduction}

Nonminimum phase system (or signal) identification is an important problem in many signal and image processing applications including data communication, seismic signal processing and optical imaging (1-4).

In this paper, we address the problem of simultaneous reconstruction of the impulse responses of four minimum or nonminimum phase FIR systems using the power spectrum and cross-trispectrum of the output sequences. We present parametric multichannel system identification methods.

$\dagger$ Portions of this paper were presented at the IEEE Signal Processing Workshop on Higher-Order Statistics, California, U.S.A., June 1993. This work is supported by TÜBITAK, Turkey. 


\section{Tankut Özgen et al.}

Recent work (5 8) on nonminimum phase multichannel system identification includes the work by Brooks and Nikias (5) who showed that three nonminimum phase systems driven by an independent and identically distributed (i.i.d.) nonGaussian process can be reconstructed simultaneously from their output crossbispectrum. Their method is a nonparametric cepstral technique which computes the complex cepstra of the impulse response sequences of the unknown systems from the third-order cross-cumulants of output sequences. Higher order statistical identification schemes which utilize complex cepstrum have been widely used in practical applications $(\mathbf{5}, \mathbf{9}-\mathbf{1 3})$. These schemes have some disadvantages when poles and zeros come close to the unit circle. Our parametric methods do not suffer from this limitation. However, they require exact knowledge of systems' orders and a theoretical analysis shows that they yield consistent parameter estimation only in a class of additive colored Gaussian noise, as well as in the additive white Gaussian noise. Experimental verification of the methods by means of simulation examples has been provided for the case of white Gaussian noise only.

The organization of the paper is as follows. In Section II we define the problem and introduce the basic concepts. In Section III we develop a least squares-type method which is based on solving a system of linear equations obtained from a relationship derived in Section II. We prove the uniqueness of the least squares solution in Section IV by devising a recursive method to determine the unknown impulse response parameters. We investigate the robustness of the new methods to additive noise in Section V. In Section VI we present simulation examples.

\section{Problem Definition}

In this section, we describe the multichannel system identification problem.

Consider the following signal model:

$$
\begin{aligned}
y_{i}(n) & =z_{i}(n)+w_{i}(n) \\
& =\sum_{k=0}^{y_{i}} h_{i}(k) x(n-k)+w_{i}(n) \quad i=1,2,3,4
\end{aligned}
$$

where $y_{i}(n)$ is the output of the $i$ th FIR system of which the impulse response is $h_{i}(n) ; q_{i}$ is the order of the $i$ th system; $w_{i}(n)$ is an additive zero-mean Gaussian noise; and $z_{i}(n)$ is the output of the $i$ th system in the absence of noise. For convenience, the impulse responses, $h_{i}(n), i=1,2,3,4$, are numbered such that $q_{1} \leqslant q_{2} \leqslant q_{3} \leqslant q_{4}$. The input sequence $x(n)$ is assumed to be an i.i.d. non-Gaussian process with $E\{x(n)\}=0, \quad E\left\{x(n) x\left(n+\tau_{1}\right)\right\}=\beta, \delta\left(\tau_{1}\right)$, $E\left\{x(n) x\left(n+\tau_{1}\right) x\left(n+\tau_{2}\right)\right\}=\beta_{3} \delta\left(\tau_{1}, \tau_{2}\right)$ and $c_{x}\left(\tau_{1}, \tau_{2}, \tau_{3}\right)=\beta_{4} \delta\left(\tau_{1}, \tau_{2}, \tau_{3}\right)$ where $c_{x}\left(\tau_{1}, \tau_{2}, \tau_{3}\right)$ denotes the fourth-order cumulants of the input $x(n)$.

In most digital communication applications the system input, $x(n)$, is derived from a signal constellation which is symmetric around the origin. Therefore the third-order cumulants of $x(n)$ are identically zero $\left(\beta_{3}=0\right)$. In such a case we use the fourth-order cumulants of the system outputs. The methods developed in this paper can be extended to the general problem of simultaneous reconstruction of an even number of FIR systems by choosing the order of the required cumulants 
to be equal to the number of systems. If the input sequence $x(n)$ is asymmetric around the origin, an odd number of systems can also be identified by using our algorithm.

Let us define $c_{1234}\left(\tau_{1}, \tau_{2}, \tau_{3}\right)$ as the fourth-order cross-cumulant sequence of the processes $\left\{y_{i}(n)\right\}_{i=1}^{4}$, i.e.

$$
\begin{aligned}
c_{1234}\left(\tau_{1}, \tau_{2}, \tau_{3}\right)= & E\left\{y_{1}\left(n+\tau_{1}\right) y_{2}\left(n+\tau_{2}\right) y_{3}\left(n+\tau_{3}\right) y_{4}(n)\right\} \\
& -E\left\{y_{1}\left(n+\tau_{1}-\tau_{2}\right) y_{2}(n)\right\} \cdot E\left\{y_{3}\left(n+\tau_{3}\right) y_{4}(n)\right\} \\
& -E\left\{y_{1}\left(n+\tau_{1}-\tau_{3}\right) y_{3}(n)\right\} \cdot E\left\{y_{2}\left(n+\tau_{2}\right) y_{4}(n)\right\} \\
& -E\left\{y_{1}\left(n+\tau_{1}\right) y_{4}(n)\right\} \cdot E\left\{y_{2}\left(n+\tau_{2}-\tau_{3}\right) y_{3}(n)\right\} .
\end{aligned}
$$

By using the fact that the fourth-order cumulants of zero-mean Gaussian noise processes are identically zero, $c_{1234}\left(\tau_{1}, \tau_{2}, \tau_{3}\right)$ can be related to the unknown impulse responses $\left\{h_{i}(n)\right\}_{i=1}^{4}$ as shown below:

$$
c_{1234}\left(\tau_{1}, \tau_{2}, \tau_{3}\right)=\beta_{4} \sum_{k=0}^{q_{4}} h_{1}\left(k+\tau_{1}\right) h_{2}\left(k+\tau_{2}\right) h_{3}\left(k+\tau_{3}\right) h_{4}(k) .
$$

The cross-trispectrum, $C_{1234}\left(\omega_{1}, \omega_{2}, \omega_{3}\right)$, of the output processes, $\left\{y_{i}(n)\right\}_{i=1}^{4}$, is defined as the three-dimensional Fourier transform of the cross-cumulant sequence, $c_{1234}\left(\tau_{1}, \tau_{2}, \tau_{3}\right)$. From (3), it follows that

$$
C_{1234}\left(\omega_{1}, \omega_{2}, \omega_{3}\right)=\beta_{4} H_{1}\left(\omega_{1}\right) H_{2}\left(\omega_{2}\right) H_{3}\left(\omega_{3}\right) H_{4}\left(-\omega_{1}-\omega_{2}-\omega_{3}\right)
$$

where $H_{i}(\omega)$ is the Fourier transform of the system impulse response $h_{i}(n)$.

We also need the second-order cumulant sequence, $s(\tau)=E\left\{z_{4}(n) z_{4}(n+\tau)\right\}$, of the noise free output sequence, $z_{4}(n)$. The power spectrum, $S(\omega)$, of $z_{4}(n)$ is

$$
S(\omega)=\beta_{2} H_{4}(\omega) H_{4}(-\omega) .
$$

\subsection{A fundamental relationship}

In this subsection, we derive a relationship between the second- and fourth-order cumulants. This relationship is the basis of our multichannel system identification method.

By multiplying both sides of Eq. (4) by $H_{4}\left(\omega_{1}+\omega_{2}+\omega_{3}\right)$ and using (5) we get

$$
H_{4}\left(\omega_{1}+\omega_{2}+\omega_{3}\right) C_{1234}\left(\omega_{1}, \omega_{2}, \omega_{3}\right)=\varepsilon H_{1}\left(\omega_{1}\right) H_{2}\left(\omega_{2}\right) H_{3}\left(\omega_{3}\right) S\left(\omega_{1}+\omega_{2}+\omega_{3}\right)
$$

where $\varepsilon=\beta_{4} / \beta_{2}$. By taking the inversc Fouricr transform of both sides of (6) we obtain the following relationship :

$$
\sum_{i=0}^{y_{4}} h_{4}(i) c_{1234}\left(\tau_{1}-i, \tau_{2}-i, \tau_{3}-i\right)=\varepsilon \sum_{i=0}^{q_{1}} h_{1}(i) h_{2}\left(\tau_{2}-\tau_{1}+i\right) h_{3}\left(\tau_{3}-\tau_{1}+i\right) s\left(\tau_{1}-i\right)
$$

which relates the impulse responses, $\left\{h_{i}(n)\right\}_{i=1}^{4}$, to the second-order cumulants, $s(n)$, of the sequence $z_{4}(n)$ and the fourth-order cross-cumulants, $c_{1234}\left(\tau_{1}, \tau_{2}, \tau_{3}\right)$, 


\section{Tankut Özgen et al.}

of the output sequences, $\left\{y_{i}(n)\right\}_{i=1}^{4}$. This relationship is the four-channel version of an equation used in some parametric system identification techniques $(11,14)$. Equation (7) is very important because it allows us to estimate the impulse responses, $\left\{h_{i}(n)\right\}_{i=1}^{4}$, by solving an overdetermined system of linear equations.

\section{Least Squares (LS) Solution}

In this section, we develop a least squares method for reconstructing the impulse response sequences, $\left\{h_{i}(n)\right\}_{i=1}^{4}$, from the second-order cumulants and the fourthorder cross-cumulants by using Eq. (7). First, we assume without loss of generality that $\left\{h_{i}(n)\right\}_{i=1}^{4}$ is scaled such that $h_{i}(0)=1, i=1,2,3,4$. Then, Eq. (7) can be arranged as follows:

$$
\begin{aligned}
c_{1234}\left(\tau_{1}, \tau_{2}, \tau_{3}\right)=\varepsilon \sum_{i=0}^{q_{1}} h_{1}(i) h_{2}\left(\tau_{2}-\tau_{1}+i\right) & h_{3}\left(\tau_{3}-\tau_{1}+i\right) s\left(\tau_{1}-i\right) \\
& -\sum_{i=1}^{q_{4}} h_{4}(i) c_{1234}\left(\tau_{1}-i, \tau_{2}-i, \tau_{3}-i\right) .
\end{aligned}
$$

By concatenating (8) for $\left(\tau_{1}, \tau_{2}, \tau_{3}\right) \in S$ where $S$ is a region which is described below, we obtain the following overdetermined system of linear equations :

$$
\mathbf{d}=\mathbf{M r}
$$

where

$$
\begin{aligned}
& \mathbf{r}=\left[h_{4}(1) \ldots h_{4}\left(q_{4}\right) \varepsilon \varepsilon h_{1}(1) \ldots \varepsilon h_{1}\left(q_{1}\right) \varepsilon h_{2}(1) \ldots \varepsilon h_{2}\left(q_{2}\right) \varepsilon h_{3}(1) \ldots \varepsilon h_{3}\left(q_{3}\right)\right. \\
& \varepsilon h_{1}(1) h_{2}(1) \ldots \varepsilon h_{1}\left(q_{1}\right) h_{2}\left(q_{2}\right) \varepsilon h_{1}(1) h_{3}(1) \ldots \varepsilon h_{1}\left(q_{1}\right) h_{3}\left(q_{3}\right) \varepsilon h_{2}(1) h_{3}(1) \ldots \\
& \left.\varepsilon h_{2}\left(q_{2}\right) h_{3}\left(q_{3}\right) \varepsilon h_{1}(1) h_{2}(1) h_{3}(1) \ldots \varepsilon h_{1}\left(q_{1}\right) h_{2}\left(q_{2}\right) h_{3}\left(q_{3}\right)\right]^{\mathrm{T}}
\end{aligned}
$$

is a $\left(q_{4}+\left(q_{1}+1\right)\left(q_{2}+1\right)\left(q_{3}+1\right)\right)$ column vector, $\mathbf{d}=\left[c_{1234}\left(\tau_{1}, \tau_{2}, \tau_{3}\right)\right.$ : $\left.\left(\tau_{1}, \tau_{2}, \tau_{3}\right) \in S\right]^{\mathrm{T}}$ is an $N\left(q_{1}, q_{2}, q_{3}, q_{4}\right)$ column vector, and $\mathbf{M}$ is a matrix of size $N\left(q_{1}, q_{2}, q_{3}, q_{4}\right) \times\left(q_{4}+\left(q_{1}+1\right)\left(q_{2}+1\right)\left(q_{3}+1\right)\right)$ of which the entries are determined according to (8). $N\left(q_{1}, q_{2}, q_{3}, q_{4}\right)$ is the number of points in the region $S$ which is determined as follows. It follows from (3) that $c_{1234}\left(\tau_{1}, \tau_{2}, \tau_{3}\right)$ is nonzero for $-q_{4} \leqslant \tau_{1} \leqslant q_{1},-q_{4} \leqslant \tau_{2} \leqslant q_{2}$ and $-q_{4} \leqslant \tau_{3} \leqslant q_{3}$. Hence, the left hand side of (7), $\sum_{i=0}^{q_{4}} h_{4}(i) c_{1234}\left(\tau_{1}-i, \tau_{2}-i, \tau_{3}-i\right)$, is nonzero for $-q_{4} \leqslant \tau_{1} \leqslant q_{1}+q_{4}$, $-q_{4} \leqslant \tau_{2} \leqslant q_{2}+q_{4}$, and $-q_{4} \leqslant \tau_{3} \leqslant q_{3}+q_{4}$. In addition, we should maintain that the $h_{2}\left(\tau_{2}-\tau_{1}+i\right) h_{3}\left(\tau_{3}-\tau_{1}+i\right)$ term at the right hand side of (7) is nonzero, yielding $0 \leqslant \tau_{2}-\tau_{1}+i \leqslant q_{2}, 0 \leqslant \tau_{3}-\tau_{1}+i \leqslant q_{3}$ for $i=0,1,2, \ldots, q_{1}$. This leads to $-q_{1} \leqslant \tau_{2}-\tau_{1} \leqslant q_{2}$ and $-q_{1} \leqslant \tau_{3}-\tau_{1} \leqslant q_{3}$. Thus, the region $S$ is defined by the following set,

$$
\begin{array}{r}
S=\left\{\left(\tau_{1}, \tau_{2}, \tau_{3}\right):-q_{4} \leqslant \tau_{1} \leqslant q_{1}+q_{4},-q_{4} \leqslant \tau_{2} \leqslant q_{2}+q_{4},-q_{4} \leqslant \tau_{3} \leqslant q_{3}+q_{4},\right. \\
\left.-q_{1} \leqslant \tau_{2}-\tau_{1} \leqslant q_{2},-q_{1} \leqslant \tau_{3}-\tau_{1} \leqslant q_{3}\right\} .
\end{array}
$$

By counting the number of points in this region, we obtain the size of the column vector $\mathbf{d}, N\left(q_{1}, q_{2}, q_{3}, q_{4}\right)$, as 


$$
\begin{aligned}
& N\left(q_{1}, q_{2}, q_{3}, q_{4}\right)=q_{1}\left(q_{1}+1\right)\left(2 q_{1}+1\right) / 3+\left(q_{2}+q_{3}+2\right) q_{1}\left(q_{1}+1\right) \\
& +2\left(q_{1}+1\right)\left(q_{2}+1\right)\left(q_{3}+1\right)+\left(2 q_{4}-q_{1}-1\right)\left(q_{1}+q_{2}+1\right)\left(q_{1}+q_{3}+1\right) .
\end{aligned}
$$

The least squares solution of the overdetermined system of linear equations given by $(9)$ is

$$
\mathbf{r}=\left(\mathbf{M}^{\mathrm{T}} \mathbf{M}\right)^{-1} \mathbf{M}^{\mathrm{T}} \mathbf{d} .
$$

$h_{4}(1), h_{4}(2), \ldots, h_{4}\left(q_{4}\right)$ can then be determined as the first $q_{4}$ elements of the vector r. The other impulse response coefficients $\left\{h_{i}(n)\right\}_{i=1}^{3}$ can directly be obtained by dividing the corresponding element of $\mathbf{r}$ by $r\left(q_{4}+1\right)$, which is $\varepsilon$.

However, directly obtained results could be inaccurate due to measurement noise and estimation errors. In that case, we identify $\left\{h_{i}(n)\right\}_{i=1}^{3}$, by using a method (11) that is based on the singular value decomposition (SVD). This method exploits all the available information provided by the vector $r$ except the information contained in the terms $\varepsilon h_{1}(i) h_{2}(j) h_{3}(k)$.

We form three matrices $\mathbf{R}\left[\mathbf{h}_{1}, \mathbf{h}_{2}\right], \mathbf{R}\left[\mathbf{h}_{1}, \mathbf{h}_{3}\right], \mathbf{R}\left[\mathbf{h}_{2}, \mathbf{h}_{3}\right]$ from the vector $\mathbf{r}$ as follows:

$$
\mathbf{R}\left[\mathbf{h}_{\mathbf{i}}, \mathbf{h}_{\mathbf{j}}\right]=\varepsilon\left[\begin{array}{ccccc}
1 & h_{j}(1) & h_{j}(2) & \ldots & h_{j}\left(q_{j}\right) \\
h_{i}(1) & h_{i}(1) h_{j}(1) & h_{i}(1) h_{j}(2) & \ldots & h_{i}(1) h_{j}\left(q_{j}\right) \\
h_{i}(2) & h_{i}(2) h_{j}(1) & h_{i}(2) h_{j}(2) & \ldots & h_{i}(2) h_{j}\left(q_{j}\right) \\
\vdots & \vdots & \vdots & \ddots & \vdots \\
h_{i}\left(q_{i}\right) & h_{i}\left(q_{i}\right) h_{j}(1) & \ldots & \ldots & h_{i}\left(q_{i}\right) h_{j}\left(q_{j}\right)
\end{array}\right]
$$

where $i, j=1,2,3$ and $i \neq j$. The matrix $\mathbf{R}\left[\mathbf{h}_{\mathbf{i}}, \mathbf{h}_{\mathbf{j}}\right]$ is of rank one and can be written in the following form

$$
\mathbf{R}\left[\mathbf{h}_{\mathbf{i}}, \mathbf{h}_{\mathbf{j}}\right]=\varepsilon\left[\begin{array}{c}
1 \\
h_{i}(1) \\
h_{i}(2) \\
\vdots \\
h_{i}\left(q_{i}\right)
\end{array}\right]\left[\begin{array}{lllll}
1 & h_{j}(1) & h_{j}(2) & \ldots & h_{j}\left(q_{j}\right)
\end{array}\right] .
$$

The unknown impulse response sequences $h_{i}(n)$ and $h_{j}(n)$ can be identified from $\mathbf{R}\left[\mathbf{h}_{\mathbf{i}}, \mathbf{h}_{\mathbf{j}}\right]$ using the singular value decomposition, i.e.

$$
\mathbf{R}\left[\mathbf{h}_{\mathrm{i}}, \mathbf{h}_{\mathrm{j}}\right]=\mathbf{Z V \mathbf { U } ^ { \mathrm { T } }}
$$

where $\mathbf{V}$ is a $\left(q_{i}+1\right)$ by $\left(q_{j}+1\right)$ matrix which has a special diagonal form. The diagonal elements of $\mathbf{V}$ are the singular values of $\mathbf{R}\left[\mathbf{h}_{\mathrm{i}}, \mathbf{h}_{\mathrm{j}}\right]$. The columns of the orthogonal matrix $\mathbf{Z}, \mathbf{z}_{1}, \mathbf{z}_{\mathbf{2}}, \ldots, \mathbf{z}_{\mathbf{q}_{\mathbf{i}}+1}$, are the left singular vectors of $\mathbf{R}\left[\mathbf{h}_{\mathbf{i}}, \mathbf{h}_{\mathbf{j}}\right]$ and the columns of the orthogonal matrix $\mathbf{U}, \mathbf{u}_{1}, \mathbf{u}_{2}, \ldots, \mathbf{u}_{\mathbf{q}_{j}+1}$, are the right singular vectors of $\mathbf{R}\left[\mathbf{h}_{\mathbf{i}}, \mathbf{h}_{\mathbf{j}}\right]$. Since $\mathbf{R}\left[\mathbf{h}_{\mathbf{i}}, \mathbf{h}_{\mathbf{j}}\right]$ is of rank one, it has only one nonzero singular value of which the corresponding singular vectors determine the impulse responses $h_{i}(n)$ and $h_{j}(n)$. From the properties of the SVD, it can be shown that (15)

$$
h_{i}(n)=k_{1} z_{1}(n) \quad 0 \leqslant n \leqslant q_{i}
$$

and 
M. Tankut $\ddot{O} z g e n$ et al.

$$
h_{j}(n)-k_{2} u_{1}(n) \quad 0 \leqslant n \leqslant q_{j}
$$

where $k_{1}$ and $k_{2}$ are constants chosen to scale the singular vectors, $\mathbf{z}_{1}$ and $\mathbf{u}_{1}$, so that $h_{i}(0)=h_{j}(0)=1$. The above step provides two different values for each impulse response sequence $h_{i}(n)$, since it is used twice in the matrices $\mathbf{R}\left[\mathbf{h}_{1}, \mathbf{h}_{\mathbf{2}}\right], \mathbf{R}\left[\mathbf{h}_{\mathbf{1}}, \mathbf{h}_{\mathbf{3}}\right]$, $\mathbf{R}\left[\mathbf{h}_{2}, \mathbf{h}_{3}\right]$. The arithmetic mean of them can be taken as the final result.

We should mention that the assumption that only one singular value of $\mathbf{R}\left[\mathbf{h}_{\mathbf{i}}, \mathbf{h}_{\mathbf{j}}\right]$ is nonzero is only theoretically valid. In practice, due to noise and estimation errors, there may be many nonzero singular values, but only a single dominant one. In such a case we keep the dominant singular value and its corresponding singular vectors. Also, the term "least squares" used in this section does not imply the optimality of the method in the sense of minimizing the mean-square estimation error. It refers to the least squares solution of Eq. (9).

\section{Uniqueness of the LS Solution and the Recursive Method}

The least squares method described in the previous section yields a unique (least squares) solution if the matrix $\mathbf{M}$ has full rank. In order to show that the matrix $\mathbf{M}$ is of full rank we first show that the elements of the unknown vector, $\mathbf{r}$, can uniquely be determined from (7) using a recursive algorithm. From this fact, we will be able to derive the unicity of the least squares approach.

By setting $\tau_{1}=\tau_{2}=\tau_{3}=-q_{4}$ in (7) and by using the fact that $h_{i}(0)=1$, $i=1,2,3,4$, we obtain

$$
\varepsilon=\frac{c_{1234}\left(-q_{4},-q_{4},-q_{4}\right)}{s\left(-q_{4}\right)}
$$

Similarly, by setting $\tau_{1}=-q_{4}$ only, we obtain

$$
\begin{aligned}
\varepsilon h_{2}\left(\tau_{2}+q_{4}\right) h_{3}\left(\tau_{3}+q_{4}\right)=\frac{c_{1234}\left(-q_{4}, \tau_{2}, \tau_{3}\right)}{s\left(-q_{1}\right)} \tau_{2}=-q_{4}, \ldots, q_{2}-q_{4}, \\
\tau_{3}=-q_{4}, \ldots, q_{3}-q_{4},
\end{aligned}
$$

and

$$
h_{2}\left(\tau_{2}+q_{4}\right) h_{3}\left(\tau_{3}+q_{4}\right)=\frac{c_{1234}\left(-q_{4}, \tau_{2}, \tau_{3}\right)}{8 s\left(-q_{4}\right)}=\frac{c_{1234}\left(-q_{4}, \tau_{2}, \tau_{3}\right)}{c_{1234}\left(-q_{4},-q_{4},-q_{4}\right)} .
$$

We can recover $h_{2}(n)$ and $h_{3}(n)$ by setting $\tau_{3}=-q_{4}$ and $\tau_{2}=-q_{4}$ in the above equation, i.e.

$$
h_{2}\left(\tau_{2}+q_{4}\right)=\frac{c_{1234}\left(-q_{4}, \tau_{2},-q_{4}\right)}{c_{1234}\left(-q_{4},-q_{4},-q_{4}\right)} \quad \tau_{2}=-q_{4}, \ldots, q_{2}-q_{4}
$$

and

$$
h_{3}\left(\tau_{3}+q_{4}\right)=\frac{c_{1234}\left(-q_{4},-q_{4}, \tau_{3}\right)}{c_{1234}\left(-q_{4},-q_{4},-q_{4}\right)} \quad \tau_{3}=-q_{4}, \ldots, q_{3}-q_{1} .
$$

Setting $\tau_{3}=-q_{4}$ in (7) yields 


$$
\begin{aligned}
& \varepsilon h_{1}\left(\tau_{1}+q_{4}\right) h_{2}\left(\tau_{2}+q_{4}\right)=\frac{c_{1234}\left(\tau_{1}, \tau_{2},-q_{4}\right)}{s\left(-q_{4}\right)} \tau_{1}=-q_{4}, \ldots, q_{1}-q_{4}, \\
& \tau_{2}=-q_{4}, \ldots, q_{2}-q_{4}
\end{aligned}
$$

and

$$
h_{1}\left(\tau_{1}+q_{4}\right) h_{2}\left(\tau_{2}+q_{4}\right)=\frac{c_{1234}\left(\tau_{1}, \tau_{2},-q_{4}\right)}{c_{1234}\left(-q_{4},-q_{4},-q_{4}\right)} .
$$

We can recover $h_{1}(n)$ by setting $\tau_{2}=-q_{4}$ in the above equation, as

$$
h_{1}\left(\tau_{1}+q_{4}\right)=\frac{c_{1234}\left(\tau_{1},-q_{4},-q_{4}\right)}{c_{1234}\left(-q_{4},-q_{4},-q_{4}\right)} \quad \tau_{1}=-q_{4}, \ldots, q_{1}-q_{4} .
$$

Similarly, we set $\tau_{2}=-q_{4}$ in $(7)$ and we obtain

$$
\begin{aligned}
& \varepsilon h_{1}\left(\tau_{1}+q_{4}\right) h_{3}\left(\tau_{3}+q_{4}\right)=\frac{c_{1234}\left(\tau_{1},-q_{4}, \tau_{3}\right)}{s\left(-q_{4}\right)} \tau_{1}=-q_{4}, \ldots, q_{1}-q_{4}, \\
& \tau_{3}=-q_{4}, \ldots, q_{3}-q_{4} .
\end{aligned}
$$

At this point, we compute $h_{4}(n), 1 \leqslant n \leqslant q_{4}$, as follows. We start with the assumption that $h_{4}(0)=1$. For $n=1$ to $\left\lfloor q_{4} / 2\right\rfloor,\lfloor\cdot\rfloor$ denoting the greatest integer smaller than the number, we set $\tau_{1}=-q_{4}+n, \tau_{2}=q_{2}-q_{4}+n$, and $\tau_{3}=q_{3}-q_{4}+n$ in (7) and we recursively obtain

$$
\begin{aligned}
h_{4}(n)=\frac{1}{c_{1234}\left(-q_{4}, q_{2}-q_{4}, q_{3}-q_{4}\right)}\left[\operatorname{ch}\left(q_{2}\right) h_{3}\left(q_{3}\right) s\left(-q_{4}+n\right)\right. \\
\\
\left.\quad-\sum_{i=0}^{n-1} h_{1}(i) c_{1234}\left(-q_{4}+n-i, q_{2}-q_{4}+n-i, q_{3}-q_{4}+n-i\right)\right] .
\end{aligned}
$$

By setting $\tau_{1}=q_{1}+q_{4}, \tau_{2}=q_{4}, \tau_{3}=q_{4}$ in (7),

$$
h_{4}\left(q_{4}\right)=\frac{\varepsilon h_{1}\left(q_{1}\right) s\left(q_{4}\right)}{c_{1234}\left(q_{1}, 0,0\right)} \text {. }
$$

Then, for $n=1$ to $\left\lfloor q_{4} / 2\right\rfloor$, we set $\tau_{1}=q_{1}+q_{4}-n, \tau_{2}=q_{4}-n, \tau_{3}=q_{4}-n$ in (7) and we recursively obtain

$$
\begin{aligned}
h_{4}\left(q_{4}-n\right)=\frac{1}{c_{1234}\left(q_{1}, 0,0\right)}[ & \varepsilon h_{1}\left(q_{1}\right) s\left(q_{4}-n\right) \\
& \left.-\sum_{i=0}^{n-1} h_{4}\left(q_{4}-i\right) c_{1234}\left(q_{1}-n+i,-n+i,-n+i\right)\right] .
\end{aligned}
$$

We note that $\left\lfloor q_{4} / 2\right\rfloor=q_{4} / 2$ if $q_{4}$ is even, and $\left\lfloor q_{4} / 2\right\rfloor=\left(q_{4}-1\right) / 2$ if $q_{4}$ is odd.

Finally, we are ready to recover the unknown parameters $\left\{\varepsilon h_{1}(i) h_{2}\left(\tau_{2}-\tau_{1}+i\right) h_{3}\left(\tau_{3}-\tau_{1}+i\right)\right\}$. For $n=0$ to $\left\lfloor q_{1} / 2\right\rfloor$, we set $\tau_{1}=-q_{4}+n$ in (7) and recursively compute 


$$
\begin{aligned}
\varepsilon h_{1}(n) h_{2}\left(\tau_{2}+q_{4}\right) h_{3}\left(\tau_{3}+q_{4}\right)=\frac{1}{s\left(-q_{4}\right)}\left[\sum_{i=0}^{n} h_{4}(i) c_{1234}\left(-q_{4}+n-i, \tau_{2}-i, \tau_{3}-i\right)\right. \\
\left.-\sum_{i=0}^{n-1} \varepsilon h_{1}(i) h_{2}\left(\tau_{2}+q_{4}-n+i\right) h_{3}\left(\tau_{3}+q_{4}-n+i\right) s\left(-q_{4}+n-i\right)\right],
\end{aligned}
$$

for $\tau_{2}=-q_{4}, \ldots, q_{2}-q_{4}$ and $\tau_{3}=-q_{4}, \ldots, q_{3}-q_{4}$. The above recursive formula requires the knowledge of $\left\{h_{4}(n)\right\}$ and $\left\{\varepsilon h_{2}(i) h_{3}(j)\right\}$ to compute $\left\{\varepsilon h_{1}(i) h_{2}\left(\tau_{2}-\tau_{1}+i\right) h_{3}\left(\tau_{3}-\tau_{1}+i\right)\right\}$. Now, we set $\tau_{1}=\tau_{2}=\tau_{3}=q_{1}+q_{4}$ in (7):

$$
\varepsilon h_{1}\left(q_{1}\right) h_{2}\left(q_{2}\right) h_{3}\left(q_{3}\right)=\frac{h_{4}\left(q_{4}\right) c_{1234}\left(q_{1}, q_{2}, q_{3}\right)}{s\left(q_{4}\right)} .
$$

Then, we start from $\varepsilon h_{1}\left(q_{1}\right) h_{2}\left(q_{2}\right) h_{3}\left(q_{3}\right)$ by setting $\tau_{1}=q_{1}+q_{4}-n$ in (7) for $n=0$ to $\left\lfloor q_{1} / 2\right\rfloor$, and we recursively compute

$$
\begin{array}{r}
\varepsilon h_{1}\left(q_{1}-n\right) h_{2}\left(\tau_{2}-q_{4}\right) h_{3}\left(\tau_{3}-q_{4}\right)=\frac{1}{s\left(q_{4}\right)}\left[\sum_{i=q_{4}-n}^{q_{4}} h_{4}(i) c_{1234}\left(q_{1}+q_{4}-n-i, \tau_{2}-i, \tau_{3}-i\right)\right. \\
\left.-\sum_{i=q_{1}-n+1}^{q_{1}} \varepsilon h_{1}(i) h_{2}\left(\tau_{2}-q_{1}-q_{4}+n+i\right) h_{3}\left(\tau_{3}-q_{1}-q_{4}+n+i\right) s\left(q_{1}+q_{4}-n-i\right)\right],
\end{array}
$$

for $\tau_{2}=q_{4}, \ldots, q_{2}+q_{4}$ and $\tau_{3}=q_{4}, \ldots, q_{3}+q_{4}$.

The recursive algorithm described above uses Eq. (7) only for certain values of $\tau_{1}, \tau_{2}, \tau_{3}$ to uniquely determine the unknown vector $\mathbf{r}$. Therefore it is equivalent to choosing linearly independent rows of the matrix $\mathbf{M}$ and solving the system of linear equations formed by these independent rows. It follows then that there are $q_{4}+\left(1+q_{1}\right)\left(1+q_{2}\right)\left(1+q_{3}\right)$ linearly independent rows of $\mathbf{M}$ where this number is the number of unknowns in the system of linear equations given by (9). Hence the rank of the matrix $\mathbf{M}$ is $q_{4}+\left(1+q_{1}\right)\left(1+q_{2}\right)\left(1+q_{3}\right)$. Since $\mathbf{M}$ has full rank, there is a unique least squares solution.

\section{Robustness to Additive Gaussian Noise}

In practical applications, the received signals, $\left\{y_{i}(n)\right\}_{i=1}^{4}$, are usually the noise corrupted version of the system outputs, $\left\{z_{i}(n)\right\}_{i=1}^{4}$. In this section, we consider the case where the noise terms $\left\{w_{i}(n)\right\}_{i=1}^{4}$ are Gaussian noise processes, independent of each other and $\left\{z_{i}(n)\right\}_{i=1}^{4}$ [see Eq. (1)].

For zero-mean Gaussian processes, cumulants of order greater than two are identically zero. Hence the fourth-order cumulants of $\left\{y_{i}(n)\right\}_{i=1}^{4}$ are not affected by additive Gaussian noise. However, the second-order cumulants are affected by the presence of Gaussian noise. The methods described in previous sections use the second-order cumulant sequence $s(\tau)$ of the noiseless case system output $z_{4}(n)$, instead of the second-order cumulant sequence $s_{y_{4}}(\tau)$ of $y_{4}(n)$. They are related to each other as follows : 


$$
s_{y_{4}}(\tau)=s(\tau)+s_{w_{4}}(\tau)
$$

where $s_{w_{4}}(\tau)$ is the second-order cumulant sequence of $w_{4}(n)$. In practice we can only estimate $s_{y_{4}}(\tau)$, not $s(\tau)$. It follows from (27)-(29) and (30)-(32) that the recursive method described in Section IV uses samples of $s(\tau)$ for which $q_{4}-\left\lfloor q_{4} / 2\right\rfloor \leqslant|\tau| \leqslant q_{4}$. If the second-order cumulants of the additive noise, $s_{w_{4}}(\tau)$, are nonzero for lags in the range $|\tau| \leqslant q$ where $q=q_{4}-\left\lfloor q_{4} / 2\right\rfloor-1$ the rccursive method will not be affected by the presence of noise as $s_{y_{4}}(\tau)=s(\tau)$ for $q<|\tau| \leqslant q_{4}$. Consequently, uniqueness and consistency of the LS solution will remain unaffected if the rows of the matrix $\mathbf{M}$ which contain the samples of $s_{w_{4}}(\tau)$ are removed. Both the least squares and recursive solutions are robust to additive white Gaussian noise because $s_{w^{\prime}}(\tau)$ is nonzero only for $\tau=0$.

\section{Simulation Examples}

Consider the following set of systems

$$
\begin{aligned}
& y_{1}(n)=x(n)-0.6 x(n-1)+w_{1}(n) \\
& y_{2}(n)=x(n)+0.75 x(n-1)+w_{2}(n) \\
& y_{3}(n)=x(n)+0.5 x(n-1)-1.25 x(n-2)+w_{3}(n) \\
& y_{4}(n)=x(n)-0.375 x(n-1)+0.8 x(n-2)+w_{4}(n)
\end{aligned}
$$

where the input signal, $x(n)$, is a zero mean, i.i.d. sequence with $\beta_{2}=5, \beta_{3}=0$ and $\beta_{4}=-34$. The noise terms, $\left\{w_{1}(n)\right\}_{1=1}^{4}$, are zero-mean, white Gaussian processes with variance 1 , and they are uncorrelated with each other and with the input signal $x(n)$.

In our simulation cxamples the data records $(N=2048), \quad\left\{y_{i}(n)\right\}_{i=1}^{4}$ ( $n=0,1, \ldots, 2047)$, were generated by the above set of systems. The impulse response coefficients of the unknown systems were estimated by using the LS method for 100 outpul realizations for the noise-free case where noise processes, $\left\{w_{i}(n)\right\}_{i=1}^{4}$, are eliminated in the signal model, as well as the noisy case. The mean value and the standard deviation for each impulse response coefficient were computed over 100 realizations. For the noisy case, rows of the coefficient matrix $\mathbf{M}$ which contain the value, $s_{y_{4}}(0)$, were removed. Experimental results are presented in Tables I and II. It is observed that the mean values are not significantly different for the noise-free and noisy cases. However, standard deviations are slightly larger for the noisy case.

TABLE I

Reconstructed impulse response coefficients for the noise-free case

\begin{tabular}{llllrrl}
\hline & $h_{1}(1)$ & $h_{2}(1)$ & \multicolumn{1}{c}{$h_{3}(1)$} & \multicolumn{1}{c}{$h_{3}(2)$} & \multicolumn{1}{c}{$h_{4}(1)$} & \multicolumn{1}{c}{$h_{4}(2)$} \\
\hline True value & -0.6 & 0.75 & 0.5 & -1.25 & \multicolumn{1}{c}{-0.375} & \multicolumn{1}{l}{0.8} \\
Mean value & -0.6121 & 0.7307 & 0.4866 & -1.2340 & -0.3931 & 0.7863 \\
Stand. dev. & 0.0422 & 0.0380 & 0.0366 & 0.0421 & 0.0358 & 0.0173 \\
\hline
\end{tabular}


M. Tankut $\ddot{O}_{z g e n}$ et al.

TABLE II

Reconstructed impulse response coefficients for the noisy case

\begin{tabular}{lllllll}
\hline & $h_{1}(1)$ & $h_{2}(1)$ & \multicolumn{1}{c}{$h_{3}(1)$} & \multicolumn{1}{c}{$h_{3}(2)$} & \multicolumn{1}{c}{$h_{4}(1)$} & \multicolumn{1}{c}{$h_{4}(2)$} \\
\hline True value & -0.6 & 0.75 & 0.5 & -1.25 & \multicolumn{1}{c}{-0.375} & \multicolumn{1}{c}{0.8} \\
Mean value & -0.6096 & 0.7319 & 0.4863 & -1.2391 & -0.3970 & 0.7851 \\
Stand. dev. & 0.0614 & 0.0586 & 0.0552 & 0.0641 & 0.0456 & 0.0281 \\
\hline
\end{tabular}

Complex-cepstra based system identification methods produce poor results when system zeros are close to the unit circle $(5,9, \mathbf{1 0})$. Our parametric methods do not suffer from this limitation. For example, in $(34) h_{3}(n)$ and $h_{4}(n)$ have zeros at $-1.3956,0.8956$ and $0.1875 \pm i 0.8746$, respectively. Although the last three zeros are close to the unit circle, our LS method produced good estimates of them.

The new methods require exact knowledge of systems' orders. In (11) an efficient system order determination scheme was developed for single channel system identification. This scheme is based on the single channel version of our fundamental equation (7). A reliable multichannel system order estimation scheme can be developed as in (11).

A consistent behavior of the new methods has been observed in all the simulation examples tried for the case of white Gaussian noise. Numerical stability of our algorithms has not been examined in the case of colored Gaussian noise disturbance.

\section{Conclusion}

In this paper new methods for simultaneous identification of four minimum or nonminimum phase LTI FIR systems driven by an i.i.d. non-Gaussian process are presented. Our methods, a least squares (LS) method and a recursive method, are parametric and use the second-and fourth-order cumulants of the system outputs in an appropriate domain of support. The recursive method is developed to prove the uniqueness of the least squares solution. The new methods can be extended to the more general problem of simultaneous identification of three or more systems by using second-order cumulants and system output cumulants of order being equal to the number of systems to be identified. We experimentally observed that the LS method yields consistent parameter estimation in the case of white Gaussian noise.

\section{Acknowledgement}

We thank the anonymous reviewer for his careful review and helpful comments.

\section{References}

(1) L. Chapparo and L. Luo, "Identification of two-dinemsional systems using sum-ofcumulants", "Proc. 1992 International Conf. on Acoustics, Speech, and Signal Processing (ICASSP'92)", pp. V-481-484, IEEE Press, 1992. 
(2) A. T. Erdem, "A nonredundant set for the bispectrum of 2-D signals", "Proc. 1993 Int. Conf. on Acoustics, Speech, and Signal Processing (ICASSP'93)", pp. IV-188191, IEEE Press, 1993.

(3) J. M. Mendel, "Tutorial on higher-order statistics (spectra) in signal processing and system theory: theoretical results and some applications", IEEE Proc., Vol, 79, pp. 278-305, 1991.

(4) C. L. Nikias and M. R. Raghuveer, "Bispectrum estimation : a digital signal processing framework", IEEE Proc., Vol. 75, pp. 869-891, 1987.

(5) D. H. Brooks and C. L. Nikias, "The cross-bicepstrum : properties and applications for signal reconstruction and system identification", in "Proc. ICASSP-91", Toronto, pp. 3433-3436, May 1991.

(6) P. Comon, "MA identification using fourth order cumulants", Signal Processing, Vol. 26, pp. 381-388, 1992.

(7) G. B. Giannakis, Y. Inouye and J. M. Mendel, "Cumulant based identification of multichannel MA models", IEEE Trans. Automat. Control, Vol. 34, No. 7, pp. 783$787,1989$.

(8) A. Swami, G. B. Giannakis and S. Shamsunder, "A unified approach to modeling multichannel ARMA processes using cumulants", IEEE Trans. Signal Processing, in press.

(9) S. Alshebeili and A. E. Çetin, "A phase reconstruction algorithm from bispectrum", IEEE Trans. Geosci. Remote Sensing, Vol. 28, pp. 166-171, March 1990.

(10) S. A. Alshebeili, A. E. Çetin and A. N. Venetsanopoulos, "Identification of nonminimum phase MA systems using cepstral operations on slices of higher order spectra", IEEE Trans. Circuits Systems : Part II Analogy Digital Signal Processing, Vol. 39, pp. 634-637, 1992.

(11) S. A. Alshebeili, A. N. Venetsanopoulos and A. E. Çctin, "Cumulant based identification approaches for nonminimum phase FIR systems", IEEE Trans. Signal Processing, Vol. 41, No. 4, pp. 1576-1588, 1993.

(12) A. E. Cetin, "An iterative algorithm for signal reconstruction from bispectrum", IEEE Trans. Signal Processing, Vol. 39, pp. 2621-2629, 1991.

(13) R.L. Pan and C. L. Nikias, "The complex cepstrum of higher order cumulants and nonminimum phase system identification", IEEE Trans. Signal Processing, Vol. 38, pp. 186-205, 1988.

(14) J. K. Tugnait, "Approaches to FIR system identification with noisy data using higher order statistics", IEEE Trans. Acoust. Speech Signal Processing, Vol. 38, pp. 1307$1317,1990$.

(15) G. H. Golob and C. F. Van Loan, "Matrix Computations", The Johns Hopkins University Press, 1983.

Received: 4 August 1993

Accepted : 26 October 1993 